\title{
FACTORES CAUSALES DE LOS TRASTORNOS DE LA CONDUCTA ALIMENTARIA ATRIBUIDOS POR PROFESIONALES SANITARIOS CAUSAL FACTORS OF EATING DISORDERS ATTRIBUTED BY HEALTH WORKERS
}

\author{
M. Elena Gismero-GonzÁlez \\ Universidad Pontificia Comillas \\ egismero@chs.upcomillas.es
}

Cómo referenciar este artículo/How to reference this article:

Gismero-González, M. E. (2012). Factores Causales de los Trastornos de la Conducta Alimentaria atribuidos por profesionales sanitarios. Acción Psicológica, 9(2), 77-86 doi: http://dx.doi.org/10.5944/ ap.9.2.4106

\section{Resumen}

Se pretende conocer cómo valoran los profesionales sanitarios anorexia y bulimia nerviosas y obesidad, en concreto su percepción de la gravedad de estos trastornos y a qué tipo de causas los atribuyen. Quinientos ocho médicos, enfermeros o psicólogos en ejercicio, de ambos sexos, respondieron anónima y voluntariamente a un cuestionario sobre gravedad y causas atribuidas a estos trastornos. Se analizan diferencias entre los trastornos, y entre los participantes en base a sus titulaciones, edad, sexo, y experiencia profesional con estos pacientes. Los participantes consideran estos trastornos muy severos, con factores causales muy diferenciados, más vinculados a factores biológicos y hábitos de vida en la obesidad, y a problemas emocionales e influencias sociales en anorexia y bulimia nerviosas. En la bulimia nerviosa dan más importancia a los hábitos de vida y los factores endocrinos, mientras en la anorexia destacan más la autodisciplina, el ideal social de delgadez, el influjo de los amigos y la vanidad. Los participantes atribuyen a estos pacientes bastante control y responsabili- dad sobre su problema (aún más a las personas obesas). Algunos resultados sugieren que obesidad, anorexia y bulimia podrían estar vinculadas con ciertas valoraciones negativas por parte de los profesionales sanitarios.

Palabras clave: atribuciones causales, anorexia nerviosa, bulimia nerviosa, obesidad, trastornos de la conducta alimentaria.

Abstract
The aim of this study is to know how health
professionals consider anorexia nervosa, buli-
mia nervosa and obesity, specifically, their per-
ception of the severity of these disorders and
their perceived causes. Five hundred and eight
physicians, nurses and psychologists in practi-
ce, of both sexes, responded anonymously and
voluntarily to a questionnaire about severity
and perceived causes of eating disorders. We
analyze differences between the disorders, and
among the participants (on their degrees, age,
sex and professional experience with these pa-
tients). Participants consider eating disorders
as very severe, with different causal factors,


more related to biological aspects and lifestyle on obesity, and to emotional difficulties and social influences in anorexia and bulimia nervosas. In bulimia they give more importance to lifestyle and endocrine factors, while in anorexia they highlight self-discipline, the social ideal of thinness, peer influence, and vanity. Participants attribute to these patients significant control and responsibility for their problem (even more to obese patients). Some of the results suggest that obesity, anorexia and bulimia nervosas could be linked to certain negative valuations for health professionals.

Key words: causal attributions, anorexia nervosa, bulimia nervosa, obesity, eating disorders.

\section{Introducción}

Los Trastornos de la Conducta Alimentaria (TCA), y en particular la anorexia nerviosa (AN), no pueden entenderse desligados de su contexto social. Además de las posibles causas de su aparición en nuestro contexto socio-histórico, y del análisis de las motivaciones profundas y significados subyacentes de quienes lo padecen (Gismero y Godoy, 2004), se considera relevante investigar cómo se comprende, se percibe y se valora a las personas que exhiben ese patrón de comportamiento por parte de su entorno y, muy especialmente, de quienes son autoridad en temas de salud, porque ello incidirá no sólo en los significados subjetivos que adquiere para quien lo padece, sino también en hacer explícita cuál es la interpretación de ese fenómeno, las expectativas sobre quienes padecen estos trastornos, y en último término, su diagnóstico y tratamiento.

En el caso de la AN, desde que fue reconocida como entidad clínica independiente hace unos cien años, la descripción que se hace sobre ella y sus causas ofrece un relato estigmatizante de las pacientes, como mujeres enfermas que eran sólo piel, con estados mentales mórbidos. La AN se define como "síndrome psiquiátrico», y las enfermedades mentales son enfermedades estigmatizadas. Por su parte, la obesidad resulta también estigmatizada en una sociedad en la que se valora a las personas por su aspecto, siendo el modelo estético imperante la delgadez.

Algunas investigaciones sugieren que la AN puede ser una enfermedad estigmatizada por la población general, al considerarla menos dependiente de factores biológicos que otros trastornos, y en cambio verla más provocada por quienes la padecen y más bajo su control (Crisp, 2005, Crisp, Gelder, Rix, Meltzer y Rowlands, 2000; Stewart, Keel y Schiavo, 2006). El propio DSM-IV (APA, 1994) incluye como primer criterio diagnóstico para la AN el «rechazo a mantener el peso corporal igual o por encima del valor mínimo normal» (p.558); por tanto, los intentos deliberados de reducir o mantener un bajopeso son inherentes a la definición del trastorno. Este aspecto parece suscitar cierto nivel de admiración en otras personas, ya que se percibe como auto-control (e.g. Branch y Eurman, 1980), pero si la AN se atribuye a auto-control, entonces la culpa de la enfermedad recaerá más fácilmente en el individuo que la padezca. Holliday, Wall, Treasure y Weinman (2005), comparando las percepciones sobre la anorexia de personas con el trastorno con otras que no lo tenían, encontraron que estas últimas consideraban la AN significativamente más controlable, y la atribuían más a la dieta y los hábitos alimenticios. Así, como en los datos de Crisp et al. (2000), parece que la visión social de la AN es la de una enfermedad auto-inducida, y esta creencia puede disminuir la empatía y aumentar el enfado o el rechazo hacia quienes la padecen.

Las investigaciones sobre la percepción social o las posibles actitudes negativas hacia la AN se han centrado en población general. Apenas se ha estudiado la percepción que de los TCA tienen los profesionales sanitarios. Fleming y Szmukler (1992) encontraron que el $59 \%$ de una muestra de médicos y enfermeros de un hospital general afirmaban que los pacientes con AN son responsables de su situación. Brotman, Stern y Herzog (1984) informan que médicos residentes expresaban tener más sentimientos negativos (enfado, tristeza, ansiedad, estrés e indefensión) cuando trataban pacientes con AN que al tratar a otros con diabetes u obesidad, quizá debido a la percepción de que la anorexia es "voluntaria y autodestructiva» (p. 76). 
Esta tendencia a culpar a las personas con AN por su situación puede implicar importantes consecuencias negativas. En general, las personas cuya situación se considera más bajo su control tienden a provocar más enfado o rabia que pena, y a elicitar menos conductas de ayuda o apoyo que aquellas cuya situación se percibe como menos controlable por su parte (Weiner, 1993). Algunos autores han sugerido que la tendencia a atribuir las enfermedades mentales a causas psicosociales, más que a causas biológicas, se intensifica especialmente en el caso de la AN, trastorno que la población no especializada (no sanitaria) no atribuye a causas biológicas o genéticas (Crisafulli, Von Holle y Bullik, 2008; Holliday et al., 2005; Stewart et al., 2006).

El objetivo de esta investigación es conocer los estereotipos que mantienen los profesionales sanitarios (médicos, enfermeros, psicólogos) sobre AN, bulimia nerviosa (BN) y obesidad, en concreto cuál es su percepción de la gravedad de estos trastornos y a qué tipo de causas los atribuyen. Específicamente, se analizarán las diferencias en la valoración que suscitan los diferentes tipos de TCA, y se explorarán algunas diferencias encontradas en función de la titulación de los profesionales (medicina, psicología, enfermería); de algunas características personales de los participantes (sexo, edad); o de su experiencia profesional de trabajo con estos problemas.

\section{Método}

\section{Participantes}

La muestra estuvo compuesta por 508 sujetos: 379 mujeres $(74.6 \%)$ y 129 varones $(25.4 \%)$. Respecto a la Titulación, 151 sujetos eran médicos (29.7\%), 176 enfermeros (34.6\%) y 189 psicólogos $(37.2 \%)$. Entre los médicos, las especialidades más representadas eran Medicina General/ Medicina Familiar y Comunitaria (38.7\%), Psiquiatría (12\%), y Medicina Preventiva y Salud Pública (8.7\%). Los psicólogos tenían distintas orientaciones: $33 \%$ cognitivo-conductual, 14.9\% humanista, $12.8 \%$ dinámica, $12.8 \%$ sistémica, $11.2 \%$ psicoanalítica y $15.4 \%$ integradora.
La edad promedio de los participantes era 36.78 años (desviación típica=11.20). Edad mínima $=20$, y máxima $=76$. Seis sujetos no respondieron a esta pregunta (1.2\%). El total de la muestra se dividió en cinco intervalos de edad, de cara a las posteriores comparaciones: menores de 28 años (26.3\%), de 28 a 38 (32.3\%), de 38 a 48 (21.5\%), de 48 a 58 (15.3\%), y 58 o más $(4.6 \%)$.

La muestra provino de todas las comunidades españolas (excepto La Rioja), destacando Madrid (67.9\%), seguida de Andalucía (12.4\%). No interesaba tanto conocer los lugares de origen de los participantes cuanto los lugares en los que desempeñan su trabajo, dado que el objetivo es investigar qué atribuciones mantienen sobre los TCA aquellos profesionales que los puedan tratar en nuestro país. Por tanto, la pregunta que se les planteó fue "¿En qué comunidad autónoma trabaja?».

Respecto a los centros de trabajo, $52.4 \%$ de los participantes trabajan en hospitales $(\mathrm{n}=266)$, $13.8 \%$ en centros de salud $(\mathrm{n}=70), 19.1 \%$ en consulta privada $(\mathrm{n}=97)$ y $23.6 \%$ en "otros lugares» $(n=120)$. En cuanto a la experiencia en tratamiento de personas con TCA, un 39.2 ha tratado anorexias; un $30.7 \%$ bulimias, y un $50.2 \%$ ha tratado pacientes con obesidad.

\section{Variables e Instrumentos}

El cuestionario de auto-informe se compone de distintas variables:

a) Variables demográficas y ocupacionales: edad, sexo, titulación, especialidad u orientación (en su caso), comunidad autónoma donde trabaja, centro de trabajo, experiencia profesional con personas con AN, BN y obesidad.

b) Causas de AN, BN y obesidad. Separadamente para cada uno de los trastornos, los participantes debían indicar en qué medida consideraban que distintos factores contribuyen a su desarrollo mediante una escala Likert de 7 alternativas ( $1=$ no contribuye en absoluto, $7=$ es el principal factor causal). Se valoraban 11 factores en el caso de $\mathrm{AN}$ y $\mathrm{BN}$, y 9 para 
la obesidad, algunos de ellos (malos hábitos de vida, influencia de los padres, falta de apoyo social, autodisciplina) se adaptaron de Stewart et al. (2006), otros (ideal social de delgadez, factores genéticos, vanidad) tomados de Crisafulli, Von Holle y Bulik (2008), y otros (trastornos endocrinos, problemas emocionales, influencia de amigos/as, búsqueda de atención) se incluyeron ad hoc para los propósitos de esta investigación. En la obesidad no se preguntó por ideal social de delgadez ni por vanidad. Se obtuvo un Alpha de Cronbach para estas variables entre 0.70 y 0.77 .

c) Severidad. La percepción de la gravedad de AN, BN y obesidad se evaluó con una pregunta sobre cada trastorno con 7 alternativas de respuesta tipo Likert ( $1=$ no muy serio, $7=$ muy grave) .

d) Atribución de responsabilidad. La opinión de los participantes sobre el grado de responsabilidad de las personas con $\mathrm{AN}, \mathrm{BN}$ y obesidad en haber llegado a crearse estos trastornos a sí mismas se evaluó con una pregunta para cada trastorno con 5 alternativas de respuesta tipo Likert $(1=$ nada responsable, $5=$ muy culpable). Una puntuación mayor corresponde a mayor grado de culpabilización del sujeto, y por tanto, a una opinión más negativa sobre el mismo, y mayor indicador de prejuicio.

e) Auto-atribución/control. La opinión de los participantes sobre en qué grado alguien con AN, BN y obesidad podría mejorar si se lo propusiera realmente se evaluó con una pregunta para cada trastorno con 5 alternativas de respuesta tipo Likert ( 1 = nada en absoluto, 5 = podría mejorar mucho), correspondiendo una puntuación mayor a mayor grado de atribución del trastorno a quien lo padece, y en consecuencia, a una opinión más negativa y mayor indicador de prejuicio. Como la variable anterior, ésta fue adaptada de la Opinions Scale de Crisp et al. (2000).

\section{Procedimiento}

Los participantes se consiguieron a través de dos vías. Se envió a médicos, psicólogos y enfermeros, de ambos sexos, un mensaje de correo electrónico solicitando su participación, voluntaria y anónima, en una investigación sobre las actitudes de los profesionales sanitarios hacia los TCA. Se pedía como requisito que se encontraran actualmente en el ejercicio de su profesión, y se solicitaba la difusión del mensaje electrónico entre sus colegas profesionales (muestreo «bola de nieve»). Este mensaje incluía un enlace directo al cuestionario con todas las variables a estudiar, adaptado al programa Sphinx Survey 14. Se elaboró otra versión del cuestionario en papel, para difundirla entre quienes preferían responder de este modo o no disponían de correo electrónico. En este segundo caso, se recuperaron sólo un 45\% de los cuestionarios repartidos, cuyas respuestas posteriormente fueron incorporadas manualmente al programa Sphinx. Aquellos cuestionarios en papel que presentaban un número considerable de respuestas en blanco se eliminaron; entre los que dejaban algún dato sin responder se valoró individualmente la conveniencia de incluirlos o no, en función del número y relevancia de las variables. El análisis de datos se realizó con los programas Sphinx Survey 14 y SPSS versión 14.0.

\section{Resultados}

\section{Factores causales atribuidos a Anorexia Nerviosa, Bulimia Nerviosa y Obesidad}

Comparaciones entre causas atribuidas a $\boldsymbol{A N}, \boldsymbol{B N} \boldsymbol{y}$ obesidad. La tabla 1 presenta los resultados de los diversos análisis de varianza (ANOVAs) de medidas repetidas que analizan las diferencias entre las atribuciones causales de los participantes respecto al desarrollo de AN, BN y obesidad. El tamaño del efecto para la comparación de medias se calculó usando Eta al cuadrado parcial $\left(\eta_{\mathrm{p}}{ }^{2}\right)$. 
Tabla 1

ANOVAs, comparación de factores que contribuyen al desarrollo del trastorno

\begin{tabular}{lrrrrrr}
\hline \multicolumn{1}{c}{ Factores causales } & $\begin{array}{c}\text { AN (N=506) } \\
\text { Media (DS) }\end{array}$ & $\begin{array}{r}\text { BN (N=506) } \\
\text { Media (DS) }\end{array}$ & $\begin{array}{r}\text { O (N=506) } \\
\text { Media (DS) }\end{array}$ & $\boldsymbol{F}$ & $\boldsymbol{p}$ & $\begin{array}{c}\text { TE } \\
\text { (np2 ) }\end{array}$ \\
\hline Malos hábitos de vida & $3.82(1.727)$ & $4.56(1.673)$ & $6.27(.982)$ & 982.347 & $<.0001$ & .660 \\
Influencia de los padres & $4.53(1.512)$ & $4.46(1.506)$ & $5.07(1.387)$ & 54.495 & $<.0001$ & .098 \\
Trastornos endocrinos & $3.16(1.545)$ & $3.40(1.562)$ & $5.16(1.465)$ & 694.462 & $<.0001$ & .580 \\
Falta de apoyo social & $4.55(1.638)$ & $4.64(1.569)$ & $4.25(1.500)$ & 16.892 & $<.0001$ & .032 \\
Autodisciplina/falta & $4.89(1.651)$ & $4.44(1.704)$ & $5.41(1.351)$ & 42.075 & $<.0001$ & .077 \\
Factores genéticos & $2.86(1.524)$ & $2.98(1.515)$ & $4.98(1.443)$ & 724.579 & $<.0001$ & .591 \\
Problemas emocionales & $6.13(.996)$ & $6.17(.964)$ & $5.28(1.274)$ & 191.785 & $<.0001$ & .276 \\
Influencia de amigos/as & $5.36(1.293)$ & $5.03(1.446)$ & $3.48(1.583)$ & 599.047 & $<.0001$ & .545 \\
Busca atención & $4.74(1.569)$ & $4.66(1.662)$ & $2.93(1.572)$ & 510.450 & $<.0001$ & .503 \\
\hline
\end{tabular}

Para cada factor, mayores medias indican que se considera que el factor contribuye en mayor medida al desarrollo del trastorno.

Como se observa, los participantes consideran factores causales mucho más relevantes en el caso de la obesidad que en AN y BN los malos hábitos de vida, factores genéticos, trastornos endocrinos, y en menor grado la influencia de los padres y la falta de autodisciplina. Los participantes dan mucha mayor importancia como factores causales de AN y BN, comparadas con obesidad, a la influencia de los amigos, la búsqueda de atención, los problemas emocionales, y en menor medida, a la falta de apoyo social.

\section{Comparaciones entre causas atribuidas a} $\boldsymbol{A N} \boldsymbol{y} \boldsymbol{B N}$. Las diferencias entre AN y BN en factores causales atribuidos por los participantes se analizaron mediante contrastes de medias para cada una de las causas. Los participantes dan más peso en el desarrollo de la $\mathrm{AN}$ a la autodisciplina $(\mathrm{t}=5.570, p<.0001, \mathrm{~d}=.27)$, el ideal social de delgadez $(\mathrm{t}=9.870, p<.0001$, $\mathrm{d}=.24)$, la influencia de amigos/as $(\mathrm{t}=6.735$, $p<.0001, \mathrm{~d}=.24)$ y la vanidad $(\mathrm{t}=4.136$, $p<.0001, \mathrm{~d}=.13)$, mientras que en el desarrollo de $\mathrm{BN}$ destacan los malos hábitos de vida $(\mathrm{t}=12.438, p<.0001, \mathrm{~d}=.43)$ y los trastornos endocrinos $(\mathrm{t}=4.089, p<.0001, \mathrm{~d}=.15)$.

Factores causales de AN. Las causas que según los participantes contribuyen más al desarrollo de la AN son el ideal social de delgadez. $(\mathrm{M}[\mathrm{DS}]=6.13$ [1.02]) y los problemas emocionales $(\mathrm{M}[\mathrm{DS}]=6.12[1.0])$, seguidos de la in- fluencia de amigos/as, auto-disciplina, búsqueda de atención, falta de apoyo social, influencia de los padres, vanidad y hábitos de vida. Consideran factores etiológicos menos importantes en la AN la genética y los trastornos endocrinos (ninguna de las dos llega al punto medio 3.5). El conjunto de estos ítems presenta un Alpha de Cronbach de 0.70.

Comparando las valoraciones de las causas según el sexo de los participantes, el contraste de medias indica que las mujeres dan más importancia $(\mathrm{M}=6.20)$ que los hombres $(\mathrm{M}=5.93)$ al papel del ideal social de delgadez $(\mathrm{t}=2.64, p=.009, \mathrm{~d}=.26)$.

Respecto a la edad de los participantes, el ANOVA arroja diferencias significativas en 4 de las variables estudiadas, que el grupo menor de 28 años considera factores más importantes que otros grupos de edad: trastornos endocrinos (más que los otros 4 grupos de edad, $F[4,496]=7.047, p<.0001)$, falta de apoyo social (más que los mayores de 58, $F[4,496]=4.647, p=.001$ ), autodisciplina (más que los de 38 a 48, $F[4.496]=3.249, p=.012$ ), e ideal social de delgadez (más que los mayores de 58, $F[4,497]=3.195, p=.013)$.

Atendiendo a la experiencia profesional con pacientes con AN, quienes les han tratado dan menos importancia al ideal social de delgadez $(\mathrm{t}=3.29, p=.001, \mathrm{~d}=0.29)$, la influencia de los 
amigos/as $(\mathrm{t}=3.42, p=.001, \mathrm{~d}=0.30)$ y la falta de apoyo social $(\mathrm{t}=2.41, p=.016, \mathrm{~d}=0.22)$.

Factores causales de BN. Los participantes destacan como causa más relevante de $\mathrm{BN}$ los problemas emocionales $(\mathrm{M}[\mathrm{DS}]=6.17$ [0.96]), seguidos, en orden decreciente, del ideal social de delgadez, influencia de amigos/ as, búsqueda de atención, falta de apoyo social, malos hábitos de vida, influencia de los padres, autodisciplina, y, en menor medida, vanidad. Como en la AN, trastornos endocrinos y factores genéticos no alcanzan el punto medio de importancia. Este segundo conjunto de ítems presenta un Alpha de Cronbach de 0.77.

Comparando las causas de $\mathrm{BN}$ atribuidas por los participantes según su sexo, las mujeres dan significativamente más peso al ideal social de delgadez $(\mathrm{t}=4.58, p<.0001, \mathrm{~d}=0.53)$, la influencia de amigos/as $(\mathrm{t}=3.52, p<.0001$, $\mathrm{d}=0.36)$ y a problemas emocionales $(\mathrm{t}=2.14$, $p=.033, \mathrm{~d}=0.22$ ).

Atendiendo a la edad de los participantes, el ANOVA arroja 3 diferencias significativas en las variables estudiadas, que el grupo menor de 28 años considera factores más relevantes que otros grupos de edad: falta de apoyo social (más que los de 38 a 48 y que los mayores de $58, F[4,495]=4.568, p=.001)$; ideal social de delgadez (más que los mayores de 58, $F[4,495]=4.549, p=.001)$ e influencia de amigos (más que los de 38 a 48 y que los mayores de $58, F[4,493]=4.891, p=.001)$.

Quienes han tratado pacientes con $\mathrm{BN}$ dan significativamente menos peso al ideal social de delgadez $(\mathrm{t}=3.82, p<.0001, \mathrm{~d}=0.39)$, la influencia de amigos/as $(\mathrm{t}=2.96, p=.003, \mathrm{~d}=0.28)$, la vanidad $(\mathrm{t}=2.82, p=.005, \mathrm{~d}=0.27)$, la búsqueda de atención $(\mathrm{t}=2.32, p=.021, \mathrm{~d}=0.22)$, la falta de autodisciplina $(\mathrm{t}=2.28, p=.023, \mathrm{~d}=0.22)$ y la falta de apoyo social $(\mathrm{t}=2.60, p=.040, \mathrm{~d}=0.20)$.

Factores causales de Obesidad. Como principal factor causal de obesidad destaca los malos hábitos de vida, seguido de falta de autodisciplina, problemas emocionales, trastornos endocrinos, influencia de los padres, genética y falta de apoyo social. En la obesidad pesan menos como factores causales la influencia de los amigos/as y la búsqueda de atención. Este conjunto de ítems obtiene un Alpha de Cronbach de 0.74 .

Las mujeres dan más importancia que los hombres en la obesidad a los trastornos endocrinos $(\mathrm{t}=2.89, p=.004, \mathrm{~d}=.29)$, la influencia de los padres $(\mathrm{t}=2.60, p=.009, \mathrm{~d}=.27)$, la falta de apoyo social $(\mathrm{t}=2.42, p=.016, \mathrm{~d}=.23)$, los factores genéticos $(\mathrm{t}=2.16, p=.031, \mathrm{~d}=.22)$, y a los malos hábitos de vida $(\mathrm{t}=2.11, p=.037, \mathrm{~d}=.23)$.

Respecto a las edades de los participantes, el ANOVA arroja diferencias significativas en todas las variables, excepto en falta de autodisciplina y en influencia de los amigos. Las diferencias encontradas en el resto de factores causales de obesidad son las siguientes: el grupo de mayores de 58 años considera menos importante los hábitos de vida que los grupos de menos de 28 , de 38 a 48 y de 38 a 48 años $(F[4,497]=4.372, p=.002)$; y también dan menor peso a la influencia de los padres que todos los demás grupos de edad $(F[4,495]=6.231$, $p<.0001)$; los menores de 28 años dan más importancia a los trastornos endocrinos que los de 48 a 58 y que los mayores de 58 , y los de 28 a 38 también dan más peso a este factor que los mayores de $58(F[4,495]=7.012, p<.0001)$; los menores de 28 años también dan más importancia a los factores genéticos que los grupos de 48 a 58 y más de $58(F[4,496]=10.905$, $p<.0001)$; y el grupo de 48 a 58 años da más importancia al papel de los problemas emocionales que los de 28 a 38 años $(F[4,496]=$ 3.605, $p=.007$ ).

Los profesionales que han tratado pacientes obesos (el $50.2 \%$ de la muestra) dan significativamente más importancia a los hábitos de vida $(\mathrm{t}=2.89, p=.004, \mathrm{~d}=.25)$ y menos a los trastornos endocrinos $(\mathrm{t}=3.50, \mathrm{p}=.001, \mathrm{~d}=0.31)$ y a la búsqueda de atención $(\mathrm{t}=2.13, p=.032$, $\mathrm{d}=0.19$ ).

Severidad. Los participantes consideran el trastorno más grave de los tres la AN (M [DS] = 6.64 [0.66], comparada con la BN (M [DS] = $6.32[0.85], \mathrm{t}=5.97, p<.001, \mathrm{~d}=.42)$ y con la obesidad (M [DS] $=6.13$ [0.9], $\mathrm{t}=9.31, p<.001$, $\mathrm{d}=.64)$. A su vez, la $\mathrm{BN}$ se considera más grave que la obesidad $(\mathrm{t}=3.32, p=.001, \mathrm{~d}=.22)$. 
Las mujeres perciben más grave que los hombres la AN $(\mathrm{t}=3.26, p=.001, \mathrm{~d}=.40)$, la BN $(\mathrm{t}=4.91, p<.0001, \mathrm{~d}=.58)$ y la obesidad $(\mathrm{t}=$ $4.87, p<.0001, \mathrm{~d}=.53)$.

Respecto a las edades de los participantes, el ANOVA no arroja diferencias entre la percepción de gravedad de AN y $\mathrm{BN}$, pero sí en obesidad $(F[4,496]=6.939, p<.0001)$, considerándola más grave los menores de 28 años que los de 38 a 48 y que los mayores de 58 , y los situados entre 28 y 38 más grave que los mayores de 58 .

Atribución de responsabilidad. No encontramos diferencias en atribución de responsabilidad sobre el desarrollo de su trastorno hacia personas con AN (M [DS] = 3.13 [1.01]) y BN $(\mathrm{M}[\mathrm{DS}]=3.19$ [0.99]), pero sí respecto a la obesidad $(\mathrm{M}[\mathrm{DS}]=3.47[0.88])$ : los participantes consideran más responsables a las personas obesas que a aquellas con $\mathrm{AN}(\mathrm{t}=5.58, p<.001$, $\mathrm{p}=.36)$ y con $\mathrm{BN}(\mathrm{t}=4.74, \mathrm{p}<.001, \mathrm{p}=.30)$.

Según sus titulaciones, los psicólogos atribuyen a las personas obesas menor responsabilidad que médicos y enfermeros $(F[2,505]=$ $6.301, p=.002)$. No aparecen diferencias en base al sexo, edades ni experiencia profesional de los participantes en las atribuciones de responsabilidad sobre quienes padecen ninguno de los tres trastornos.

Atribución de control. Como ocurría en la atribución de responsabilidad, no aparecen diferencias entre las opiniones de los participantes sobre personas con AN (M [DS] $=3.66$ [1.06]) y $\mathrm{BN}(\mathrm{M}[\mathrm{DS}]=3.78[1.0])$, pero de nuevo, consideran que las personas obesas $(\mathrm{M}[\mathrm{DS}]=4.16[0.9])$ podrían mejorar más si se lo propusieran seriamente que las que tienen $\mathrm{AN}(\mathrm{t}=5.58, p<.001, \mathrm{~d}=.51)$ y $\mathrm{BN}(\mathrm{t}=$ 4.74, $p<.001, \mathrm{~d}=.40)$.

Por titulaciones, no hay diferencias en cuanto a la obesidad, pero sí respecto a la AN $(F[2,505]=7.233, p=.001)$ y la BN $(F[2,505]$ $=4.787, p=.009)$ : en ambos casos los médicos les atribuyen menos capacidad de control que los enfermeros y psicólogos. No hay diferencias entre los tres trastornos en base al sexo ni las edades de los participantes en esta variable. Respecto a la experiencia profesional de los participantes, quienes han diagnosticado AN consideran en menor grado que quienes no los han diagnosticado que estos pacientes podrían mejorar si se lo propusieran $(\mathrm{t}=2.93, p=.004, \mathrm{~d}=.26)$.

Relaciones entre atribución de responsabilidad y de capacidad de control. Tal como esperábamos, existe relación entre atribuir a las personas con cada trastorno estudiado responsabilidad en haber llegado a desarrollarlo y verles más capaces de mejorar si se lo proponen. Hemos encontrado las siguientes correlaciones entre ambas variables: para AN: $.236(p$ $<.0001)$; para $\mathrm{BN}: .281(p<.0001)$; para la obesidad: $.244(p<.0001)$.

\section{Discusión}

La muestra obtenida en este estudio es bastante amplia, de edades y procedencias diversas, con un porcentaje de sexos que ha permitido establecer adecuadamente diferencias, y con una representación proporcionada de los tres colectivos que se pretendían estudiar, así como un considerable porcentaje de profesionales con experiencia con estos trastornos, con lo que presumiblemente pueda ofrecer una visión de la percepción sobre los TCA de los profesionales sanitarios en España razonablemente generalizable. Dado que la participación era voluntaria y anónima, cabe suponer que las personas más negativamente inclinadas hacia estos trastornos hayan tenido menor interés en participar.

El conjunto de la muestra considera extremadamente graves los tres trastornos estudiados, el más grave la AN, seguido de la BN. Los enfermeros valoran aún más la gravedad de los tres que los médicos, así como las mujeres, comparadas con los hombres. Por edades, los jóvenes consideran más grave la obesidad que los de mayor edad. Así pues, los profesionales sanitarios tienen una percepción de los TCA como trastornos muy severos. Valoran la AN como el trastorno más grave de los tres, sugiriendo que son conscientes de la morbilidad, mortalidad y complicaciones médicas asociadas con este trastorno.

Con respecto a los factores causales, los profesionales sanitarios estudiados dan significativamente más importancia al papel de los 
factores biológicos (genéticos y endocrinos) como causas de obesidad que de AN y BN. Así, valoran la obesidad como un trastorno más vinculado a aspectos «biológicos» y a malos hábitos de vida, con influencias más marcadas de los padres y la falta de autodisciplina, que AN y $\mathrm{BN}$, que consideran más relacionadas con factores «psicológicos» (problemas emocionales, auto-disciplina, vanidad) y «socio-relacionales» (ideal social de delgadez, influencia de amigos, búsqueda de atención, falta de apoyo social).

Matizando más las comparaciones entre AN y BN, los participantes sitúan la $\mathrm{BN}$ algo más a "medio camino», ya que en ella dan más peso al papel de malos hábitos de vida y trastornos endocrinos, mientras que en la AN destacan más la autodisciplina, el ideal social de delgadez, la influencia de amigos/as y la vanidad. Por tanto, de los tres trastornos es la AN el que parece considerarse más producido por influencias externas que recaen sobre una persona con ciertas características que le hacen más vulnerable a ellos: problemas emocionales, autodisciplina y vanidad.

La falta de importancia atribuida a factores biológicos como causas de AN sorprende, tratándose de una muestra de profesionales de la salud: sus resultados se aproximan más a la imagen que tienen de la AN muestras de población general de otras investigaciones, que enfatizan más causas no físicas en este trastorno (e.g. Holliday et al., 2005; Lee, 1997; Stewart et al. 2006; Smith et al., 1986). Stewart et al. (2006) citan otros estudios que aportan datos sobre el importante papel de los factores biológicos en la etiología de la $\mathrm{AN}$, y de tasas de predisposición genética similares a las de esquizofrenia o asma; nuestros resultados sugieren que los sanitarios en España no son conscientes de estos datos. Parece necesario seguir investigando más a fondo sobre las diversas causas de la AN, incluyendo los aspectos biológicos; sin menoscabar la importancia obvia de los factores sociales, el hecho es que éstos no afectan por igual a todas las mujeres. Quizá haberse considerado durante mucho tiempo un "problema de adolescentes» pueda haber llevado a trivializarlo en algunos casos. La imagen que tiene nuestra muestra de las anoréxicas, como personas con problemas emocionales, autodisciplina y vanidad, que se dejan influir por el ideal social de delgadez, parece apoyar en cierto modo esta visión.

Los sanitarios estudiados atribuyen un grado de responsabilidad media (tendente a alta) a las personas que padecen estos tres trastornos por haber llegado a creárselos a sí mismos. Pero es la obesidad el trastorno que, comparativamente, atrae más atribuciones de responsabilidad, así como también de control: se atribuye a quien tiene obesidad mayor capacidad de mejorar si se lo propusiera seriamente. $P a$ rece que, a pesar de ser el trastorno más vinculado a causas "biológicas", al considerarse también que el tipo de hábitos de vida de quien lo padece es un factor determinante en su génesis, se culpa más a quien ha llegado a desarrollarlo. Y son los médicos y enfermeros, más que los psicólogos, quienes responsabilizan en mayor medida a las personas obesas.

Considerábamos que un mayor grado de atribución de responsabilidad a las personas por su problema sería un indicador de prejuicio hacia ellas, y es razonable suponer, a partir de los resultados obtenidos (y a falta de otros datos comparativos) que obesidad, $B N$ y $A N$ son trastornos vinculados con ciertas valoraciones negativas por parte de los profesionales sanitarios. No obstante, Crisafulli et al. (2008) obtuvieron, en una muestra de mujeres estudiantes de enfermería en Estados Unidos, puntuaciones sensiblemente inferiores a las que hemos obtenido en esta investigación en las variables responsabilización y control. El grado de responsabilidad sobre el problema se asocia también, como era previsible, a la valoración del grado de control de la persona sobre su problema en los tres trastornos estudiados. A mayor percepción de control por parte del sujeto, mayor atribución de responsabilidad.

Las puntuaciones medias de los participantes han sido algo más elevadas en la variable control que en la de responsabilidad atribuida; quizá la afirmación de que consideran que alguien con AN/BN/obesidad podría mejorar si se lo propusiera seriamente resulta más deseable socialmente que afirmar que alguien con ese problema es responsable de haber llegado a provocárselo a sí mismo. En cualquier caso, la inclusión de la va- 
riable control ha permitido constatar otras diferencias: como en responsabilidad, se atribuye mayor control sobre su problema a las personas obesas que a las que tienen $\mathrm{AN}$ y $\mathrm{BN}$; los médicos son quienes atribuyen menor capacidad de control sobre su problema a personas con AN y $\mathrm{BN}$ de los tres colectivos profesionales; y finalmente, los profesionales que han diagnosticado AN consideran en menor grado que quienes no los han diagnosticado que estos pacientes podrían mejorar si se lo propusieran.

Nuestros resultados son consistentes con los de otras investigaciones sobre AN, donde se constata que la gente tiende a creer que la AN es una enfermedad auto-provocada por quien la padece (Crisp et al., 2000; Fleming y Szmukler, 1992), y tiende a pensar que es controlable en un grado mucho mayor que quienes se ven afectados por ella (Holliday et al., 2005). Stewart et al. (2006), con una muestra de población general, encuentran también que a las personas con $\mathrm{AN}$ se las considera más responsables por su problema y más capaces de mejorar si se lo proponen que a quienes padecen esquizofrenia y asma, y concluyen que la percepción de control -por la que las personas con AN reciben a menudo admiración- parece incrementar el estigma al culpar de su problema a las personas con AN. Fleming y Szmukler (1992), en la única investigación sobre actitudes de profesionales sanitarios sobre los TCA que conocemos, obtuvieron resultados similares a los nuestros: sus médicos y enfermeras veían a los pacientes con TCA tan responsables de provocar su enfermedad como a quienes toman sobredosis recurrentes. Parece necesario entender qué motivos llevan a una persona a embarcarse en un comportamiento de ayuno continuado y deliberado, pero no tan voluntario ni tan controlable como puede parecer. Simplificar estos trastornos, considerándolos más centrados en la obsesión por no comer o intentar mantener un peso bajo, es trivializarlos: se requiere un conocimiento más en profundidad de las dinámicas psicopatológicas implicadas en ellos, y desde diversas perspectivas, tal como hemos sugerido en anteriores ocasiones (Gismero, 2001, 2004; Gismero y Godoy, 2004).
Respecto al sexo de los profesionales, hemos constatado que, en conjunto, las mujeres consideran los TCA problemas más severos (AN y $\mathrm{BN})$ y dan más importancia al ideal social de delgadez en el desarrollo de $A N$ y $B N$ (y en la $\mathrm{BN}$ también a la influencia de los amigos y a los problemas emocionales). Podemos concluir que las mujeres sanitarias están más sensibilizadas hacia estos trastornos que sus colegas varones, y que son más conscientes de, o valoran más intensamente, las presiones socioculturales que afectan diferencialmente más a su sexo.

Atendiendo a las diferencias entre los tres colectivos profesionales, los médicos consideran algo menos graves $\mathrm{AN}$ y $\mathrm{BN}$ que enfermeros y psicólogos, atribuyen menor capacidad de control sobre su trastorno a quienes tienen AN y BN; y dan en conjunto menos importancia a los problemas emocionales como desencadenantes de obesidad y BN. Algunas de las diferencias encontradas podrían explicarse por la formación específica que reciben, pero, dado que la mitad de los varones de la muestra $(49.6 \%)$ corresponden a la titulación de Medicina, quizá la menor percepción de gravedad de los TCA se explique mejor por el sexo de los profesionales que por su formación.

Los sanitarios estudiados son muy conscientes de la gravedad de AN y BN, como hemos comprobado, y a la vez, los consideran trastornos poco vinculados a causas biológicas. Esto último ha sido sugerido como un aspecto que podría provocar emociones más negativas hacia quienes padecen un problema determinado. El reconocimiento de los estereotipos que mantienen sobre los TCA los profesionales sanitarios puede ayudarles a ellos y a sus pacientes, contribuyendo a evitar que sus interacciones se vean afectadas (detección, derivación, hospitalizaciones, abordaje terapéutico, consejo a familiares) y aumentar nuestro conocimiento general sobre estos serios problemas, ya que son estos profesionales quienes transmiten a la población general una visión de la $\mathrm{AN}$, incidiendo, directa e indirectamente, en la visión de las personas con AN sobre sí mismas, y en su búsqueda de ayuda especializada. Como sugieren Stewart et al (2008), la presencia de cierto estigma hacia las personas con AN puede actuar como una barrera para la búsqueda de tratamiento. 


\section{Referencias}

American Psychiatric Association. (1994) DSM-IV. Manual diagnostico y estadístico de los trastornos mentales. [Diagnostic and Statistic Manual of Mental Disorders] Barcelona, España: Masson.

Branch, C. H. y Eurman, L. J. (1980). Social attitudes toward patients with anorexia nervosa. American Journal of Psychiatry, 137, 631-632.

Brotman, A., Stern, T. y Herzog, D. (1984). Emotional reactions of house officers to anorexia nervosa, diabetes and obesity. International Journal of Eating Disorders, 3, 71-77. doi: 10.1002/1098-108X(198422)3:4<71::AIDEAT2260030409>3.0.CO;2-O

Crisafulli, M. A., Von Holle, M. S. y Bulik, C. M. (2008). Attitudes towards anorexia nervosa: the impact of framing on blame and stigma. International Journal of Eating Disorders, 41, 4, 333-339. doi: 10.1002/eat.20507

Crisp, A. (2005). Stigmatization of and discrimination against people with eating disorders including a report of two national surveys. European Eating Disorders Review, 13, 147-152.

Crisp, A. H., Gelder, M. G., Rix, S, Meltzer, H. I. y Rowlands, O. J. (2000). Stigmatization of people with mental illnesses. British Journal of Psyquiatry, 177, 4-7.

Fleming, J. y Szmukler, G. I. (1992). Attitudes of medical professionals towards patients with eating disorders. Australian and New Zealand Journal of Psychiatry, 26, 436-443. doi: 10.3109/00048679209072067

Gismero E. (2001). Evaluación del autoconcepto, la satisfacción con el propio cuerpo y las habilidades sociales en la anorexia y bulimia nerviosas. [Evaluation of self-concept, satisfaction with one's body and social skills in anorexia nervosa and bulimia nervosa]. Clínica y Salud, 12(3), 289-304.
Gismero E. (2004). Más allá de la apariencia: aportaciones a la comprensión de la anorexia nerviosa a la luz de la historia. [Beyond appearance: contributions to the understanding of anorexia nervosa in the light of history]. A tu Salud, año XII, 46, 2-9.

Gismero E. y Godoy A. (2004). Una aproximación constructivista a la anorexia nerviosa: ¿tienen sentido los síntomas? [A constructivist aproach to anorexia nervosa: do symtoms have meaning?] Revista de Psicoterapia, XV (58-59), 5-31.

Holliday, J., Wall, E., Treasure, J. y Weinman, J. (2005). Perceptions on illness in individuals with anorexia nervosa: a comparison with lay men and women. International Journal of Eating Disorders, 37, 50-56. doi: 10.1002/eat.20056

Lee, S. (1997). How lay is lay? Chinese students' perceptions of anorexia nervosa in Hong Kong. Social Science and Medicine, 44, 491-502. doi: 10.1016/S0277-9536(96)00168-2

Smith, M. C., Pruitt, J. A., Mann, L. M. y Thelen, M. H. (1986). Attitudes and knowledge regarding bulimia and anorexia nervosa. International Journal of Eating Disorders, 5, 545-553. doi: 10.1002/1098-108X(198603)5:3<545::AIDEAT2260050311>3.0.CO;2-M

Stewart, M. C., Schiavo, R. S., Herzog, D. B. y Franko, D. L. (2008). Stereotypes, prejudice and discrimination of women with anorexia nervosa. European Eating Disorders Review, 16(4), 311-318. doi: 10.1002/erv.849

Stewart, M. C., Keel, P. K. y Schiavo, R. S. (2006). Stigmatization of anorexia nervosa. International Journal of Eating Disorders, 39(4), 320-325. doi: 10.1002/eat.20262

Weiner, B. (1993). On sin versus sickness. A theory of perceived responsibility and social motivation. American Psychologist, 48, 957-965. doi: 10.1037//0003-066X.48.9.957 